\title{
Análisis de diversidad genética en cerdo criollo san pedreño utilizando datos de pedigrí
}

\section{Analysis of genetic diversity in san pedreño indigenous swine using pedigree data}

\author{
Ricardo Jose Ocampo-Gallego ${ }^{1 *}$ (], Jaime Alberto Tobón-Castaño ${ }^{1}$, Piedad Yanneth Martínez-Oquendo ${ }^{1}$, Edison \\ Julián Ramírez-Toro1 [0, Carlos Edmundo Lucero-Casanova ${ }^{1}$ \\ ${ }^{1}$ Corporación Colombiana de Investigación Agropecuaria - AGROSAVIA. Centro de Investigación el Nus, CP. 250047, Corregimiento San \\ José del Nus, municipio de San Roque, Antioquia, Colombia. \\ *Autor de correspondencia: rocampo@agrosavia.co
}

Artículo científico recibido: 19 de noviembre de 2018 aceptado: 23 de marzo de 2019

RESUMEN. Colombia cuenta con tres razas de cerdos criollos (San Pedreño, Zungo y Casco de Mula), los cuales se encuentran en el país hace 500 años aproximadamente y están en riesgo de desaparecer debido a sus reducidas poblaciones y al uso preferente de razas foráneas mejoradas en los sistemas de producción. Para conservar las razas criollas se crearon los bancos de germoplasma, cuyas poblaciones se han mantenido como núcleos cerrados. El objetivo del estudio fue realizar un análisis de pedigrí para caracterizar la estructura genética de la población de cerdos San Pedreño del banco de germoplasma. Información genealógica colectada entre 2008-2017 se analizó para estimar el intervalo generacional, nivel de integridad del pedigrí, consanguinidad y evolución a través del tiempo. Los animales nacidos entre 2015 y 2017 se seleccionaron como población de referencia para estimar el tamaño efectivo poblacional y parámetros derivados de la probabilidad del origen genético. El intervalo generacional promedio para el periodo estudiado fue de 2.13 años y la consanguinidad general de $4.66 \%$. Para la población de referencia, la consanguinidad fue del $6.73 \%$ y el tamaño efectivo poblacional de 25.31, en tanto que el número efectivo de fundadores y ancestros fue de 13 y 7 individuos, respectivamente; lo que ha conllevado a cuellos de botella en la población. Los resultados indican que para mantener la variabilidad genética y reducir los niveles de consanguinidad es necesario incluir individuos no emparentados de otras poblaciones.

Palabras clave: Banco de germoplasma, consanguinidad, genealogía, intervalo generacional, porcino.

ABSTRACT. Colombia has three indigenous swine breeds (San Pedreño, Zungo and Casco de Mula), which are in the country approximately 500 years ago and are at risk of disappearing due to their reduced populations and the preferential use of improved foreign breeds in the production systems. In order to preserve the indigenous breeds, germplasm banks were created, whose populations have been maintained as closed nucleus. The objective of this study was to perform a pedigree analysis to characterize the genetic structure of the San Pedreño pig population of the germplasm bank. Genealogical information collected between 2008-2017 was analyzed to estimate the generational interval, pedigree completeness level, consanguinity and its evolution over time. The animals born between the years 2015 and 2017 were selected as the reference population to estimate the effective population size and parameters derived from the probability of gene origin. The average generational interval for the period studied was 2.13 years and the general consanguinity was $4.66 \%$. For the reference population, the consanguinity was $6.73 \%$ and the effective population size was 25.31 , while the effective number of founders and ancestors was 13 and 7 individuals, respectively, which has led to bottlenecks in the population. The results indicate that in order to maintain genetic variability and reduce inbreeding levels it is necessary to include unrelated individuals from other populations.

Key words: Germplasm bank, consanguinity, genealogy, generational interval, porcine.

\section{INTRODUCCIÓN}

El programa de conservación de una raza en peligro de extinción debe considerar como punto de partida y elemento fundamental para la formación y mantenimiento de poblaciones puras con el fin 


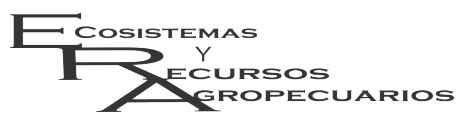

Ocampo-Gallego et al.

Diversidad genética en cerdos criollos colombianos

Ecosist. Recur. Agropec.

6(17):333-341,2019

de conservar la variabilidad genética existente en la raza/especie (Santana 2017). Tradicionalmente la diversidad genética se define como la variedad de alelos o diferentes genotipos presentes en una población, los cuales reflejan las diferencias morfológicas, fisiológicas y de comportamiento que se pueden apreciar entre los individuos y poblaciones (Frankham et al. 2002).

El mantenimiento de la diversidad genética es de gran importancia en la crianza de animales debido a que la heterocigosidad y la diversidad alélica se pueden perder a una tasa acelerada cuando se cuenta con poblaciones pequeñas, cerradas y seleccionadas (Sheikhlou y Abbasi 2016). En Colombia, la gran variedad de regiones agroclimáticas presentes ha favorecido el desarrollo de especies animales y vegetales autóctonas, entre las cuales se encuentran incluidas tres razas porcinas, tres ovinas y siete bovinas (Ocampo et al. 2017). Entre las razas porcinas criollas, se cuenta con las razas Zungo en la costa Atlántica, Casco de Mula en los Ilanos orientales y San Pedreño en el departamento de Antioquia y el viejo Caldas, los cuales tuvieron su origen a partir del cerdo ibérico español que fue introducido por los conquistadores españoles en 1493 (Oslinger 2006).

Los cerdos criollos colombianos se caracterizan por presentar altas tasas reproductivas, tolerancia a enfermedades parasitarias y gran adaptación a las condiciones del trópico que les permiten producir y reproducirse, contrarrestando factores adversos como la alimentación deficiente, escasez de agua, enfermedades y manejo precario; por todas estas características, los cerdos criollos poseen un papel muy importante en la economía y alimentación campesina (Oslinger 2006). Pero a pesar de todas las bondades y extraordinaria capacidad de adaptación que poseen, las razas criollas porcinas se encuentran en riesgo de desaparecer, debido al uso preferente de razas mejoradas foráneas en los sistemas de producción porcícolas, estimándose el número de ejemplares de la raza San Pedreño en el país en menos de 200 individuos, además se presume que las poblaciones presentan altas tasas de consanguinidad y alto grado de parentesco, lo que situaría a la raza en estado de riesgo de acuerdo a las categorías definidas por la FAO (FAO 2013).

La Corporación Colombiana de Investigación Agropecuaria AGROSAVIA es la institución encargada de conservar y fomentar el uso de los recursos genéticos autóctonos del país, y para ello dispone del Sistema de Bancos de Germoplasma de la Nación para la Alimentación y la Agricultura (SBGNAA), proyecto financiado por el Ministerio de Agricultura y Desarrollo Rural en convenio con el Instituto Colombiano Agropecuario ICA (Martínez et al. 2012). Actualmente, la población de cerdos San Pedreño del banco de germoplasma está compuesto por 125 individuos, distribuidos en 6 grupos familiares que se manejan bajo el sistema de apareamiento circular cíclico (Nomura y Yonezawa 1994). Debido a que se trata de un núcleo cerrado, se puede tener un aumento considerable de la consanguinidad, lo cual puede conllevar a efectos negativos sobre las características o aptitudes productivas y reproductivas del animal (Martínez et al. 2012).

El impacto de esta estrategia de manejo reproductivo sobre la evolución de la diversidad genética puede evaluarse en el nivel genealógico a través del pedigrí de los animales utilizando criterios basados en la probabilidad de identidad por descendencia de genes y criterios basados en probabilidades de origen genético (Sheikhlou y Abbasi 2016). Por lo anterior el objetivo de este estudio fue analizar la información de pedigrí de los cerdos San Pedreño del banco de germoplasma en el centro de investigación el Nus de AGROSAVIA para cuantificar la diversidad y estructura genética poblacional en términos de niveles de consanguinidad, tamaño efectivo de la población, intervalo generacional, coeficientes de parentesco y contribución de los fundadores a la población actual, para definir recomendaciones sobre la estrategia de conservación y políticas de reproducción.

\section{MATERIALES Y MÉTODOS}

\section{Información utilizada}

Se utilizó información colectada de los libros de campo de los cerdos San Pedreño (Figura 1) del SBGNAA en el centro de investigación el Nus de AGROSAVIA (San Roque, Antioquia, Colombia) 


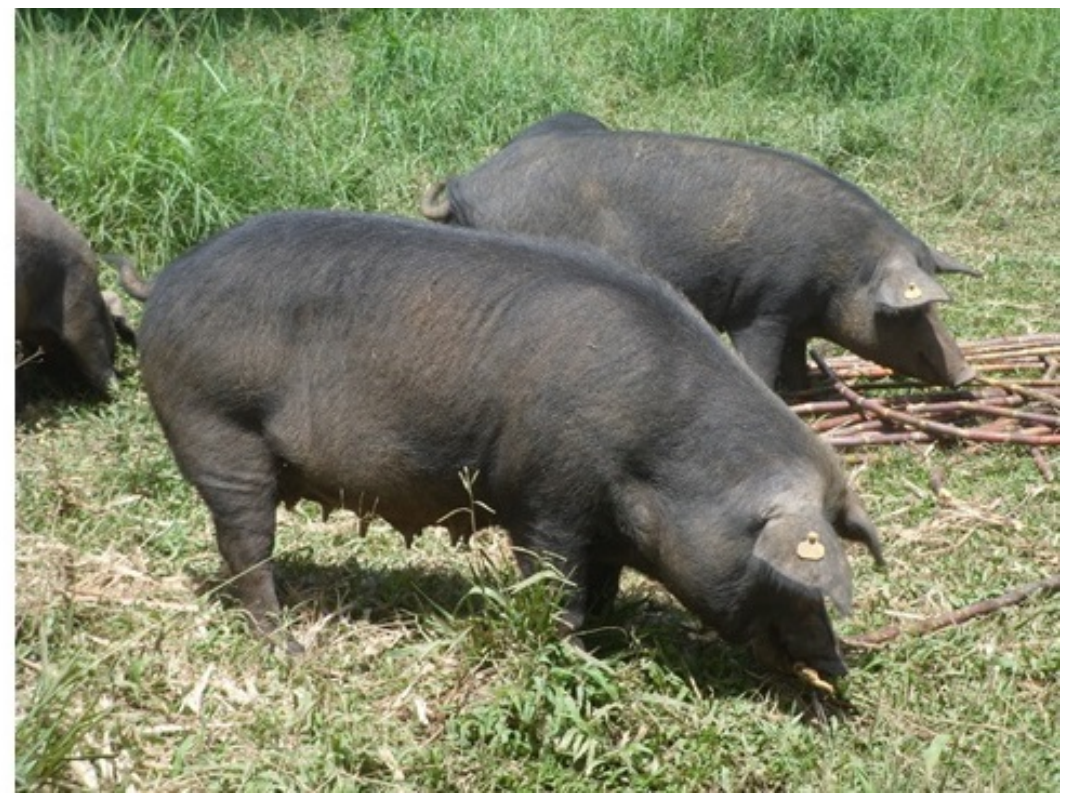

Figura 1. Ejemplares de la raza San Pedreño.

entre los años 2008 y 2017. Cada registro completo en la base de datos estuvo conformado por el código de identificación del individuo, sexo, fecha de nacimiento e identificación del padre y madre. La base de datos incluyó 1794 animales (901 hembras y 893 machos). Todos los parámetros poblacionales se estimaron considerando la población de referencia definida como los animales que nacieron entre 2015 y 2017 ( $n=664)$, lo que equivale a los animales nacidos durante el último intervalo generacional.

\section{Intervalo generacional}

El intervalo generacional se define como la edad promedio de los padres cuando nace su descendencia. Este parámetro se calculó para las cuatro vías genéticas posibles: padre-hijo, padre-hija, madre-hijo y madre-hija. Mientras que el intervalo de generación promedio la población se define como el promedio de las cuatro vías.

\section{Nivel de integridad del pedigrí}

La integridad de pedigrí expresa cuanta información generacional en promedio se dispone de cualquier individuo del pedigrí. El número de generaciones para determinado individuo se puede calcular de tres maneras: número de generaciones completas dado como el número de generaciones en que se conocen ambos padres; número de generaciones máximas (número total de generaciones posibles si los padres son conocidos o desconocidos) y número de generaciones equivalentes, que se calculó de la siguiente manera:

$$
E q G i=\sum\left(\frac{1}{2}\right)^{n}
$$

Donde: $\mathrm{n}$ es el número de generaciones en las que el animal está separado de cada antepasado con un registro conocido (Maignel et al. 1996).

La comparación entre los números de generaciones completas y máximas permite verificar la integridad de la información de pedigrí, por lo que los números cercanos para ambos indican la existencia de pocos animales desconocidos en todo el pedigrí.

\section{Probabilidad de origen genético}

La contribución genética esperada de cada fundador de la población de referencia se definió como la probabilidad de que un gen tomado al azar dentro de la población de referencia proceda de un 


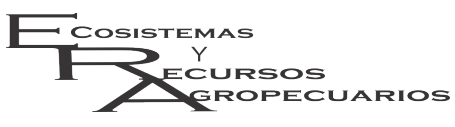

fundador determinado. El número total de fundadores $(f)$ contiene información limitada con respecto a la diversidad genética de la población porque algunos fundadores se han utilizado más intensamente y, por lo tanto, contribuyen más a la población de referencia que otros fundadores. Para tener en cuenta esto, se estimó el número efectivo de fundadores $(f e)$. Este número se definió como el número de fundadores que contribuyen por igual y que se espera que produzcan la misma diversidad genética en las poblaciones bajo estudio (Lacy 1989). La estimación de este parámetro permite medir el equilibrio de las contribuciones genéticas entre los fundadores. El número efectivo de fundadores se calculó como:

$$
f e=\frac{1}{\sum_{k=1}^{f} q_{k}^{2}}
$$

Donde: $f$ representa el número de fundadores y $q_{k}$ es la contribución genética del fundador $k$ th a la población de referencia (Gutiérrez y Goyache 2005). Para un número total dado de fundadores, cuanto más equilibradas sean sus contribuciones genéticas esperadas, mayor será el número efectivo de fundadores.

Los ancestros principales (fundadores o no) fueron estimados con el método propuesto por Boichard et al. (1997). La contribución marginal esperada $\left(q_{j}\right)$ de cada ancestro principal $(j)$ se calculó como su contribución genética esperada, independientemente de las contribuciones de los otros antepasados. El número efectivo de antepasados $(f a)$, es el número mínimo de antepasados (incluidos los fundadores y los no fundadores) necesarios para explicar la diversidad genética de la población, se estimó de la siguiente manera:

$$
f a=\frac{1}{\sum_{j=1}^{a} q_{j}^{2}}
$$

Donde: $q_{j}$ es la contribución marginal de un antepasado $j$; en otras palabras, la contribución genética realizada por un antepasado que no fue explicada por otros ancestros elegidos previamente (Gutiérrez y Goyache 2005). Calculado de esta manera, $f a$ es una medida de la diversidad genética que explica los cuellos de botella recientes y, por lo
Diversidad genética en cerdos criollos colombianos

Ecosist. Recur. Agropec. 6(17):333-341,2019

tanto, explica en parte la pérdida de la diversidad alélica desde la fundación de la población (Sørensen et al. 2005).

La proporción entre el número efectivo de ancestros y fundadores $\left(\frac{f a}{f e}\right)$ ayuda a identificar si el uso de reproductores resultó en un cuello de botella genético. Los valores inferiores a uno indican que el número efectivo de fundadores es mayor que el de los antepasados, lo que indica un posible cuello de botella genético en la población.

\section{Parámetros poblacionales relacionados con el co- eficiente de consanguinidad Coeficiente de parentesco promedio}

El coeficiente de parentesco promedio de un individuo es la probabilidad de que un alelo seleccionado al azar de la población pertenezca a un determinado animal. Este coeficiente puede interpretarse como la representación del animal en el pedigrí (Goyache et al. 2003, Gutiérrez y Goyache 2005). De acuerdo con Gutiérrez et al. (2003), este parámetro se calcula como el promedio de los coeficientes en la fila de la matriz de parentesco del numerador correspondiente al individuo.

\section{Coeficiente de consanguinidad}

La consanguinidad $(F)$ es la probabilidad de que un individuo tenga dos alelos idénticos por descendencia, para su calculo se utilizó el algoritmo desarrollado por Meuwissen y Luo (1992). El aumento de consanguinidad por generación se calculó de la siguiente manera:

$$
\Delta F=\frac{F_{t}-F_{t-1}}{1-F_{t-1}}
$$

Donde: $F_{t}$ y $F_{t-1}$ son la consanguinidad promedio en las generaciones $t_{t h}$ y $t_{t h-1}$, respectivamente.

\section{Tamaño efectivo de la población}

El tamaño efectivo de la población $\left(N_{e}\right)$ es el número de individuos que contribuyeron de manera efectiva dejando descendientes, transmitiendo genes a la siguiente generación y manteniendo la diversidad genética (Gutiérrez y Goyache 2005). El tamaño efectivo poblacional se calculó de la siguiente manera 


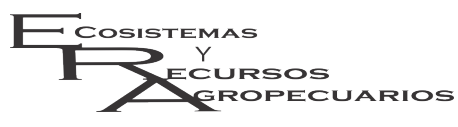

Ocampo-Gallego et al.
Diversidad genética en cerdos criollos colombianos

Ecosist. Recur. Agropec.

6(17):333-341,2019

para la población de referencia:

$$
N_{e}=\frac{1}{2 \Delta F}
$$

Donde: $\Delta F$ es el aumento relativo de endogamia por generación.

\section{Software utilizado}

La integridad del pedigrí, el intervalo generacional, los parámetros derivados de la probabilidad de origen génico, los parámetros poblacionales relacionados con el coeficiente de consanguinidad y el índice de conservación genética se estimaron con el programa ENDOG 4.8 (Gutiérrez y Goyache 2005).

\section{RESULTADOS}

La evolución de los individuos registrados por año de nacimiento es presentado en la Figura 2. A través de los años de estudio se puede observar que el número nacimientos ha variado considerablemente, presentándose la mayor cantidad de nacimientos en el 2011 con 289 nacimientos, en tanto que el menor número de nacimientos se registró en el 2008 con 12 nacimientos pero a partir de dicho año se presentó un incremento progresivo del número de nacimientos por año. El intervalo generacional promedio fue de 2.13 años. En cuanto al intervalo generacional promedio en las 4 vías posibles fueron: padre-hijo $=2.01$ años, padre-hija $=$ 2.05 años, madre-hijo $=2.19$ años, madre-hija $=2.27$ años. Presentando el menor intervalo generacional la vía padre-hijo(a).

\section{Nivel de integridad del pedigrí}

El análisis de integridad del pedigrí reveló que de los 1794 registros, el número de animales con ambos padres conocidos fue de 1754 individuos, lo que corresponde al $97.77 \%$ de la población total, presentando un solo progenitor desconocido 10 animales, lo que corresponde al $0.56 \%$ de los registros. Mientras que el análisis de integridad del pedigrí para toda la población reveló 8 generaciones máximas, 4 generaciones completas y 5.18 generaciones equivalentes (Tabla 1).

\section{Probabilidad de origen genético}

En la población de referencia se identificaron 22 animales que fueron identificados como fundadores, mientras que el número efectivo de fundadores para la misma población fue de 13 individuos. El número de ancestros que explica el $100 \%$ de la variabilidad genética de la población de referencia es de 18 individuos (fundadores o no), en tanto que solo 3 individuos explicaron el $50 \%$ de la variabilidad genética. Respecto a la proporción del número efectivos de ancestros y fundadores en la población de referencia fue 0.54 y por lo tanto inferior a 1, lo que indica la ocurrencia de posibles cuellos de botella en la población (Tabla 2 ).

\section{Parámetros poblacionales relacionados con el co- eficiente de consanguinidad}

La consanguinidad promedio para todos los animales del pedigrí y la población de referencia fue de 4.66 y $6.73 \%$, respectivamente. De acuerdo con la Figura 2, la consanguinidad promedio por año para todos los animales en el pedigrí del banco de germoplasma ha venido presentado un incremento progresivo en la consanguinidad desde el 2010, siendo el 2016 el año que se presentó la consanguinidad histórica más elevada en la población con un $7.80 \%$.

En la Tabla 3 se observa que la consanguinidad, el número de animales consanguíneos y el parentesco aumentó a lo largo de las generaciones completas, siendo el incremento de la consanguinidad por generación completa del $2.25 \%$. De igual manera se observa que en la cuarta generación completa todos los animales de la población bajo estudio son consanguíneos. El tamaño efectivo de la población $\left(N_{e}\right)$ fue de 25.31, valor que está por debajo del tamaño efectivo poblacional mínimo definido por la FAO de 50 individuos como nivel crítico.

\section{DISCUSIÓN}

En el banco de germoplasma de la nación colombiana los nacimientos de cerdos San Pedreño del 2008 al 2017 ha oscilado, debido a que los recursos que se destinan para el mantenimiento de los animales de los bancos de germoplasma se ha redu- 


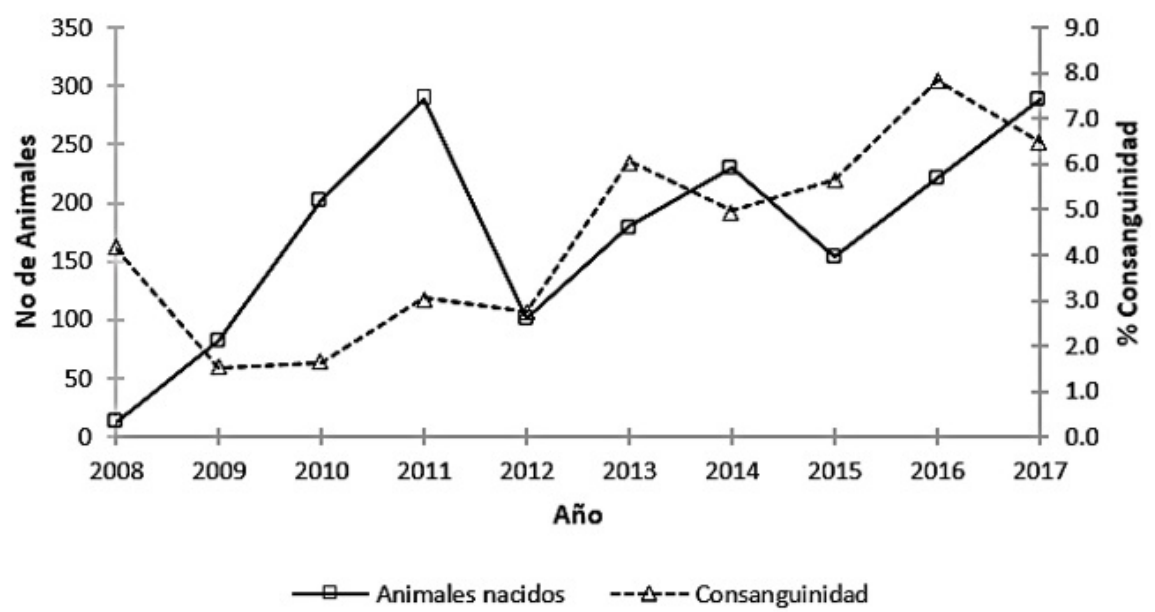

Figura 2. Evolución del número de animales registrados y consanguinidad por año de nacimiento.

Tabla 1. Estructura del pedigrí en la población de cerdos San Pedreño del banco de germoplasma entre 2008-2017.

\begin{tabular}{lc}
\hline Parámetro & Valor \\
\hline Número de Animales & 1794 \\
Número de machos & 893 \\
Número de hembras & 901 \\
Número de padres & 45 \\
Número de madres & 85 \\
Animales con progenie & 130 \\
Animales sin progenie & 1664 \\
Animales con padre y madre conocidos & 1754 \\
Animales con solo padre desconocido & 10 \\
Animales con solo madre desconocida & 0 \\
Machos con padre y madre desconocidos & 13 \\
Hembras con padre y madre desconocidos & 17 \\
Generaciones máximas & 8 \\
Generaciones completas & 4 \\
Generaciones equivalentes & 5.18 \\
\hline
\end{tabular}

Tabla 2. Resumen de parámetros calculados para la probabilidad del origen genético en la población de referencia.

\begin{tabular}{lc}
\hline Parámetro & Valor \\
\hline Número total de animales fundadores & 22 \\
Número efectivo de animales fundadores $(f e)$ & 13 \\
Número de ancestros & 18 \\
Número de ancestros que explican el $50 \%$ del pool genético & 3 \\
Número efectivo de ancestros $(f a)$ & 7 \\
Contribución del principal ancestro $(\%)$ & 18.2 \\
Relación $\left(\frac{f a}{f e}\right)$ & 0.54 \\
\hline
\end{tabular}

cido, lo que no permite mantener altas poblaciones de animales. En el 2008 el bajo número de nacimientos observados (12 lechones) obedece a que el ban- co de germoplasma se creó ese año con animales jóvenes, no aptos para la reproducción en su momento. Por otro lado, entre el 2011 y 2017 se presentó el mayor número de nacimientos (288 y 289 lechones, respectivamente), lo que permitió realizar actividades de fomento entre los productores del departamento de Antioquia encaminados a incrementar el número de cerdos criollos San Pedreño en el país.

Con respecto al intervalo generacional, cuando se estimó por las cuatro vías posibles, el intervalo generacional fue mayor para la vía de la madre-hijo(a) que cuando se calculó para la vía padre-hijo(a). El uso del sistema de apareamiento circular en el banco de germoplasma puede ser la causa de estos patrones, debido a que la selección de las hembras de reemplazo tiene lugar después de la selección de los machos, lo que resulta en intervalos generacionales mayores para las hembras que los machos. El intervalo generacional promedio fue de 2.13 años, valor que es menor que lo reportado por Santana et al. (2017) para el cerdo criollo cubano de 2.16 años y los intervalor de $2.43,2.47$ y 2.49 años reportado por Posta et al. (2016) para las razas criollas húngaras de cerdos Mangalica rubio, rojo y vientre de golondrina. Pero al compararlo con razas mejoradas utilizadas en los sistemas de producción porcícolas, el intervalo generacional promedio encontrado para la raza San Pedreño es considerablemente mayor que 


\begin{tabular}{|c|c|c|c|c|c|}
\hline $\begin{array}{l}\text { Generaciones } \\
\text { completas }\end{array}$ & $\begin{array}{c}\text { No. de } \\
\text { animales }\end{array}$ & $\begin{array}{c}\text { F promedio } \\
(\%)\end{array}$ & $\begin{array}{c}\% \text { de } \\
\text { consanguíneos }\end{array}$ & $\begin{array}{l}\text { F promedio para animales } \\
\text { consanguíneos }(\%)\end{array}$ & A promedio (\%) \\
\hline 0 & 40 & 0 & - & - & 4.2 \\
\hline 1 & 41 & 1.2 & 4.9 & 25 & 7.1 \\
\hline 2 & 556 & 2.2 & 21 & 10.6 & 11.2 \\
\hline 3 & 583 & 5 & 87 & 5.8 & 15.6 \\
\hline 4 & 574 & 7.3 & 100 & 7.3 & 17.3 \\
\hline
\end{tabular}

los valores de 1.60 y 2.07 años reportados por Melka y Schenkel (2010), Welsh et al. (2010) y Tang et al. (2013) para las razas Yorkshire, Duroc, Hampshire y Landrace. Lo que indica que la diferencia presentada en estas razas podría deberse a la mejora en el manejo reproductivo y la menor longevidad en estas razas. El mayor intervalo generacional en los cerdos San Pedreño del banco de germoplasma refleja los problemas relacionados con la reducción drástica en el censo de animales de esta raza en los últimos años, lo que retrasa la sustitución de los animales en la población.

Para el análisis de integridad del pedigrí, de los 1794 individuos que componen el pedigrí del banco de germoplasma, 10 animales presentaron padre desconocido y los 30 que poseen padre $y$ madre desconocidos en el pedigrí forman parte de los individuos con los que inicio el programa de conservación o los padres fundadores. Para el número de generaciones obtenidas a partir del pedigrí, no se observó una diferencia considerable entre el número de generaciones máximas (8), equivalentes (5.18) y generaciones completas (4), lo cual que indica que no hay pérdida considerable de información genealógica en los individuos del pedigrí (Danchin-Burgue et al. 2012). La falta de información en los datos genealógicos puede hacer que se subestimen los coeficientes de consanguinidad y parentesco en el promedio de la población y en los animales más antiguos.

En la probabilidad del origen genético de la población de referencia (animales nacidos entre 2015 y 2017) la proporción entre el número efectivo de ancestros y fundadores indica como los animales han contribuido al desarrollo de la población; si el número efectivo de fundadores es mayor que el número efec- tivo de ancestros, es muy posible que se hayan presentado cuellos de botella en la formación de la población (Sørensen et al. 2005). Para los cerdos San Pedreño del banco de germoplasma proporción se tuvo un valor de 0.54 , lo que indica que la población se ha visto afectada por cuellos de botella. Valores inferiores fueron encontrados por Posta et al. (2016) para las razas criollas húngaras Mangalica Rubia y Mangalica Rojo para los cuales se reportaron proporciones de ancestros y fundadores de 0.44 y 0.53 , respectivamente. Mientras que un mayor desbalance entre el número de ancestros y fundadores fueron observado en el trabajo realizado por Janssens et al. (2005) en poblaciones de las razas Landrace Belga y Pietrain con proporciones de ancestros y fundadores de 0.35 y 0.32 , respectivamente.

En lo referente a la consanguinidad promedio de todos los animales del pedigrí (4.66\%) y de la población de referencia $(6.73 \%)$, se ha presentado un incremento progresivo en los últimos años, lo que ha superado el límite recomendado por la FAO del 5\% para poblaciones cerradas (FAO 2013). En el análisis de las generaciones completas, se observó que la consanguinidad y el parentesco promedio se incrementan conforme aumenta el número de generaciones completas. El incremento de la consanguinidad por generación completa $(2.25 \%)$ es superior al 1\% recomendado por la FAO (2013), lo que indica que se puede evitar la depresión por endogamia y por consecuencia la pérdida de la variabilidad genética en la población (Ocampo y Cardona 2013, Bernardes et al. 2016, Sheikhlou y Abbasi 2016).

El $N_{e}$ estimado para la población de referencia fue de 25.31 individuos. Teniendo en cuenta que 


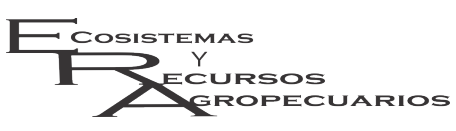

Ocampo-Gallego et al.

Diversidad genética en cerdos criollos colombianos

Ecosist. Recur. Agropec.

6(17):333-341,2019

el tamaño efectivo poblacional sugerido por la FAO (1998) de 50 individuos y lo reportado por Meuwissen (1999) quien concluyo que debido a la mutación y la deriva genética el $N_{e}$ crítico debería estar entre 50 y 100 animales. Por lo que el tamaño efectivo poblacional encontrado en el presente estudio es bajo, lo que representa una situación desfavorable para la población de cerdos del banco de germoplasma e implica la necesidad de prácticas de conservación.

\section{CONCLUSIONES}

El análisis del pedigrí es una herramienta de monitoreo del estado de la diversidad genética de las poblaciones. Con la correcta supervisión de los parámetros poblacionales se puede evitar la perdida de la diversidad genética. Para la población de cerdos San Pedreño del banco de germoplasma, que se maneja como población cerrada, sin entrada de animales ajenos al banco de germoplasma, el análisis de la información indica que la consanguinidad y el tamaño efectivo poblacional no se encuentran dentro de los valores recomendados para poblaciones de animales domésticos. Mientras que el número efectivo de fundadores es mayor que el número efectivo de ancestros, lo que ha derivado en cuellos de botella. Por lo que mantener la diversidad genética a largo plazo dependerá del esfuerzo para incrementar el tamaño efectivo de la población, equilibrar la contribución de los ancestros y evitar el apareamiento de animales emparentados para reducir la consanguinidad, por lo que es recomendado incluir individuos no emparentados de otras poblaciones.

\section{AGRADECIMIENTOS}

A la Corporación Colombiana de Investigación Agropecuaria (AGROSAVIA) y al Instituto Colombiano Agropecuario (ICA) por la financiación de los proyectos de conservación e investigación en las razas criollas colombianas a través de fondos públicos.

\section{LITERATURA CITADA}

Bernardes DA, Grossi RP, Savegnago ME, Buzanskas SB, Ramos EP, Romanzini DG, et al. (2016) Population structure of Tabapuã beef cattle using pedigree analysis. Livestock Science 187: 96-101.

Boichard D, Maignel L, Verrier É (1997) The value of using probabilities of gene origin to measure genetic variability in a population. Genetic Selection Evolution 29: 5-23.

Danchin-Burge C, Leroy G, Brochard M, Moureaux S, Verrier E (2012) Evolution of the genetic variability of eight French dairy cattle breeds assessed by pedigree analysis. Journal of Animal Breeding and Genetics 129: 206-17.

FAO (1998) Secondary Guidelines for Development of National Farm Animal Genetic Resources Management Plans, Management of Small Populations at Risk. Food Agriculture Organization, Rome, Italy. 237p.

FAO (2013) In vivo conservation of animal genetic resources. FAO Animal Production and Health Guidelines, No. 14, Rome, Italy. 270p.

Frankham R, Briscoe DA, Ballou JD (2002) Introduction to Conservation Genetics. Cambridge University Press. Cambridge. UK. 617p.

Goyache F, Gutiérrez JP, Fernández I, Gómez E, Álvarez I, Díez J, et al. (2003) Using pedigree information to monitor the genetic variability of endangered populations: the Xalda sheep breed of Asturias as an example. Journal of Animal Breeding and Genetics 120: 95-103.

Gutiérrez JP, Cañón J, Rico M (2003) Aplicación de un método modificado de cálculo del coeficiente de consanguinidad en una muestra del ganado vacuno Frison Español. Archivos de Zootecnia 39: 3-8. 
Gutiérrez JP, Goyache F (2005) A note on ENDOG: a computer program for analysing pedigrí information. Journal of Animal Breeding and Genetics 122: 172-76.

Janssens S, Depuydt J, Serlet S, Vandepitte W (2005) Genetic variability in pigs assessed by pedigree analysis: the case of Belgian Landrace NN and Pietrain in Flanders. Proc. $56^{\text {th }}$ Annual Meeting of EAAP, Session G2.39. Uppsala, Sweden. 17p.

Lacy RC (1989) Analysis of founder representation in pedigrees-founder equivalents and founder genome equivalents. Zoobiology 8: 111-23.

Maignel L, Boichard D, Verrier E (1996) Genetic variability of French dairy breeds estimated from pedigree information. Interbull 14: 49-54.

Martínez R, Vásquez RE, Gallego JL (2012) Eficiencia productiva de la raza Bon en el trópico colombiano. Corpoica, Bogotá, Colombia. 215p.

Melka M, Schenkel F (2010) Analysis of genetic diversity in four Canadian swine breeds using pedigree data. Journal of Animal Science 90: 331-340.

Meuwissen T (1999) Operation of conservation schemes, in JK Oldenbroek (ed.), Genebanks and the Conservation of Farm Animal Genetic Resources, DLO Inst. Anim. Sci. Heal, Lelystad, Netherlands. 126p.

Meuwissen TI, Luo Z (1992) Computing inbreeding coefficients in large populations. Genetic Selection Evolution 24: 305-313.

Nomura T, Yonezawa K (1994) A comparison of four systems of group mating for avoiding inbreeding. Genetic Selection Evolution 28: 141-159.

Ocampo RJ, Cardona H (2013) La endogamia en la producción animal. Revista Colombiana de Ciencia Animal 5: $465-479$.

Ocampo RJ, Martinez RA, Rocha JF, Cardona H (2017) Genetic diversity of Colombian sheep by microsatellite markers. Chilean journal of agricultural research 76: 40-47.

Oslinger A, Muñoz JE, Alvarez LA, Ariza F, Moreno F, Posso A (2006) Caracterización de cerdos criollos colombianos mediante la técnica molecular RAMs. Acta Agronomica 55: 45-50.

Posta J, Szabó P, Komlósi I (2016) Pedigree analysis of mangalica pig breeds. Annals of Animal Science 16: 701-709.

Santana I, Abeledo CM, Sánchez N (2017) Estimación de los niveles de endogamia en el centro genético núcleo del cerdo criollo cubano. AICA 9: 129-134.

Sheikhlou M, Abbasi MA (2016) Genetic diversity of Iranian Lori-Bakhtiari sheep assessed by pedigree analysis. Small Ruminant Research 141: 99-105.

Sørensen AC, Sørensen MK, Berg P (2005) Inbreeding in Danish dairy cattle breeds. Journal of Dairy Science 88: 1865-1872.

Tang G, Xue J, Lian M, Yang R, Liu T, Zeng Z, et al. (2013) Inbreeding and genetic diversity in three imported Swine breeds in China using pedigree data. Asian-Australasian Journal of Animal Science 26: 755-765.

Welsh C, Stewart T, Schwab C, Blackburn H (2010) Pedigree analysis of 5 swine breeds in the United States and the implications for genetic conservation. Journal of Animal Science 88: 1610-1618. 
